\title{
RADIATION FROM MICROSTRIP DISCONTINUITIES
}

by

M.D. Abouzahra and L. Lewin

Scientific Report No. 37

\author{
Electromagnetics Laboratory \\ Department of Electrical Engineering \\ University of Colorado \\ Boulder, Colorado 80309
}

November 1978

This project is supported in part by NSF Grant No. ENG78-09029 


\title{
RADIATION FROM MICROSTRIP DISCONTINUITIES
}

\author{
M.D. Abouzahra and L. Lewin
}

\section{Abstract}

Radically different methods of calculation by Lewin and by van der Pauw are compared. It is shown that the difference in the two sets of results is due not to the difference in methods, but to assumptions on the value of dielectric constant to be used when calculating the substrate polarization. Curves show that differences of up to $30 \%$ can arise from this source, but the differences are much smaller for the larger values of dielectric constant. 


\section{RADIATION FROM MICROSTRIP DISCONTINUITIES}

\section{M.D. Abouzahra and L. Lewin}

In an earlier paper ${ }^{(1)}$ radiation frommicrostrip discontinuities were calculated using the Poynting vector method, the fields being obtained from the strip current and the dielectric polarization beneath the strip. In order to account for leakage of the field into the air above the strip, the effective dielectric constant $\varepsilon$ was used in place of the actual constant $\varepsilon^{*}$. The substitution was made both in the propagation constant, and also in the polarization part of the calculation. The reason for doing so in the latter was that since some of the field lines leak into the air, where they give no polarization contribution, the polarization effect is reduced by an amount comparable to the velocity reduction, so it seemed reasonable to use the value $\varepsilon$ everywhere, and not just in the propagation constant.

The radiated power was calculated for a unit incident current wave and took the form

$$
P=60(k t)^{2} F(\varepsilon)
$$

$$
\text { where } \begin{aligned}
k & =2 \pi / \lambda_{0} \\
t & =\text { substrate thickness } \\
F(\varepsilon) & =\text { radiation factor }
\end{aligned}
$$


$F(\varepsilon)$ depends on the discontinuity, and takes the form

$$
F_{1}(\varepsilon)=\frac{\varepsilon+1}{\varepsilon}-\frac{(\varepsilon-1)^{2}}{2 \cdot \varepsilon^{3 / 2}} \log \frac{\varepsilon^{\frac{1}{2}}+1}{\varepsilon^{\frac{1}{2}}-1}
$$

for an open circuit, and

$$
F_{2}(\varepsilon)=1-\frac{\varepsilon-1}{2 \varepsilon^{\frac{1}{2}}} \log \frac{\varepsilon^{\frac{1}{2}}+1}{\varepsilon^{\frac{1}{2}}-1}
$$

for a matched termination.

Van der pauw (2) has given a completely different type of analysis, utilizing Fourier transforms and a rigorous treatment of the microstrip configuration, from which low frequency approximations can be obtained based on the assumption of an axial strip current uniform over the strip width. The formula for the power radiated by the open circuit differs from (2); in particular it contains both $\varepsilon$ and $\varepsilon^{*}$, and reduces to (2) in the limit for large $\varepsilon$, apart from an initial factor $\varepsilon / 2$. The latter comes essentially from referring the radiation to a peak unit voltage maximum rather than to a unit incident current wave; but the presence of both $\varepsilon$ and $\varepsilon^{*}$ elsewhere in the formula suggested that the main difference stemmed not from the radically different treatment, but from the use of $\varepsilon^{*}$ rather than $\varepsilon$ in the calculation of the contribution of the dielectric polarization to the radiated fields. To check out this latter feature the calculation of reference 1 was repeated with $\varepsilon$ replaced by $\varepsilon^{*}$ in the polarization term. The Hertzian vector consists of two components; $\Pi_{z}$ from the strip current and $\pi_{x}$ from the polarization. The latter contains a factor $(\varepsilon-1) / \varepsilon$ and if this is replaced by $\left(\varepsilon^{*}-1\right) / \varepsilon^{*}$ the following modified forms for the far-field are obtained from an open-circuited microstrip end : 


$$
\begin{aligned}
& E_{\phi}=j 120 k t \varepsilon^{\frac{1}{2}} \frac{\varepsilon^{*}-1}{\varepsilon^{*}} \frac{\cos \theta \sin \phi}{\varepsilon-\cos ^{2} \theta} \frac{e^{-j k r}}{r} \\
& E_{\theta}=-j 120 k t \varepsilon^{\frac{1}{2}}\left[\frac{\sin ^{2} \theta+\cos ^{2} \theta\left(\varepsilon^{*}-1\right) / \varepsilon^{*}}{\varepsilon-\cos ^{2} \theta}\right] \cos \phi \frac{e^{-j k r}}{r}
\end{aligned}
$$

with $r, \theta$ and $\phi$ spherical coordinates from the strip open circuit. The Poynting vector can now be constructed and integrated over an infinite hemisphere to give

$$
\begin{aligned}
\mathrm{F}_{1}\left(\varepsilon, \varepsilon^{*}\right)=\frac{2 \varepsilon}{\varepsilon^{* 2}}\{1 & +\left(\varepsilon^{* 2}-4 \varepsilon^{*}+2 \varepsilon+1\right)\left[\frac{1}{2(\varepsilon-1)}-\frac{1}{4 \varepsilon^{\frac{1}{2}}} \log \frac{\varepsilon^{\frac{1}{2}}+1}{\varepsilon^{\frac{1}{2}}-1}\right] \\
& \left.+\frac{\varepsilon^{* 2}-\varepsilon^{2}}{\varepsilon}\left[\frac{1}{2(\varepsilon-1)}+\frac{1}{4 \varepsilon^{\frac{1}{2}}} \log \frac{\varepsilon^{\frac{1}{2}}+1}{\varepsilon^{\frac{1}{2}}-1}\right]\right\}
\end{aligned}
$$

On comparison with van der Pauw's equation (21), using the interpretation for his symbols of $a=L C=\varepsilon, b=\varepsilon^{*}$, it is seen that the two results are the same, apart from the above-mentioned initial factor of $\varepsilon / 2$. Hence it may be concluded that the two methods, so very different in approach, can give essentially the same final result. Whether or not it is more appropriate to use $\varepsilon$ everywhere, as in reference 1 , is another matter, and cannot be resolved by a simple comparison of the formulas. A graph of eq. (6) is given in figure 1. The value of $\varepsilon$ ranges from $\varepsilon^{*}$ for wide stripsto $\left(\varepsilon^{*}+1\right) / 2$ for very narrow strips. It is also of interest to note that for large $\varepsilon$, eq. (6) has the asymptotic expansion

$$
\mathrm{F}_{1}\left(\varepsilon, \varepsilon^{*}\right) \sim \frac{8}{3 \varepsilon}\left[1+\frac{4}{5 \varepsilon}-\frac{1}{\varepsilon^{*}}+\cdots\right]
$$

so that the leading term $8 / 3 \varepsilon$ is identical to that obtainable from 
eq. (2). The graph of eq. (6) is given for $F_{1}\left(\varepsilon, \varepsilon^{*}\right)$ vs. $\varepsilon$ for a range of values of $\varepsilon^{*}$. It is seen that the modified formula always gives a greater value than the original, with differences of up to $30 \%$ when $\varepsilon=2, \varepsilon^{*}=3$. In consonance with eq. (7) the differences decrease greatly for larger values of $\varepsilon$.

A similar calculation can be made for the strip terminated in a matched stub. The electric field components are

$$
\begin{aligned}
& E_{\phi}=j 60 k t \varepsilon^{\frac{1}{2}}\left[\frac{\left(\varepsilon^{*}-1\right) / \varepsilon^{*}}{\varepsilon^{\frac{1}{2}}-\cos \theta}-\varepsilon^{-\frac{1}{2}}\right] \sin \phi \frac{e^{-j k r}}{r} \\
& E_{\theta}=j 60 k t \varepsilon^{\frac{1}{2}}\left[\frac{\cos \theta / \varepsilon^{*}-\varepsilon^{-\frac{1}{2}}}{\varepsilon^{\frac{1}{2}}-\cos \theta}\right] \cos \phi \frac{e^{-j k r}}{r}
\end{aligned}
$$

and the radiation factor becomes

$$
\mathrm{F}_{2}\left(\varepsilon, \varepsilon^{*}\right)=\frac{\varepsilon \varepsilon^{*^{2}}+\varepsilon^{2}-2 \varepsilon \varepsilon^{*}}{\varepsilon^{* 2}(\varepsilon-1)}-\frac{\varepsilon^{\frac{1}{2}}}{2 \varepsilon^{* 2}}\left(\varepsilon^{* 2}-2 \varepsilon^{*}+\varepsilon\right) \log \frac{\varepsilon^{\frac{1}{2}}+1}{\varepsilon^{\frac{1}{2}}-1}
$$

This possesses the asymptotic expansion

$$
\mathrm{F}_{2}\left(\varepsilon, \varepsilon^{*}\right)=\frac{2}{3 \varepsilon}\left[1+\frac{6}{5 \varepsilon}-\frac{2}{\varepsilon^{*}}+\cdots\right]
$$

and, as in the case of the open-circuit, reduces to the leading term of the unmodified formula for large $\varepsilon$.

\section{References}

1. Lewin, L., "Radiation from Discontinuities in Stripline," Proc. IEE, 1960, pp. 163-170.

2. Van der Pauw, L.J., "The Radiation of Electromagnetic Power by Microstrip Configurations," IEEE Trans. MTT, Vo1. 25, Sept. 1977, pp.719725. 


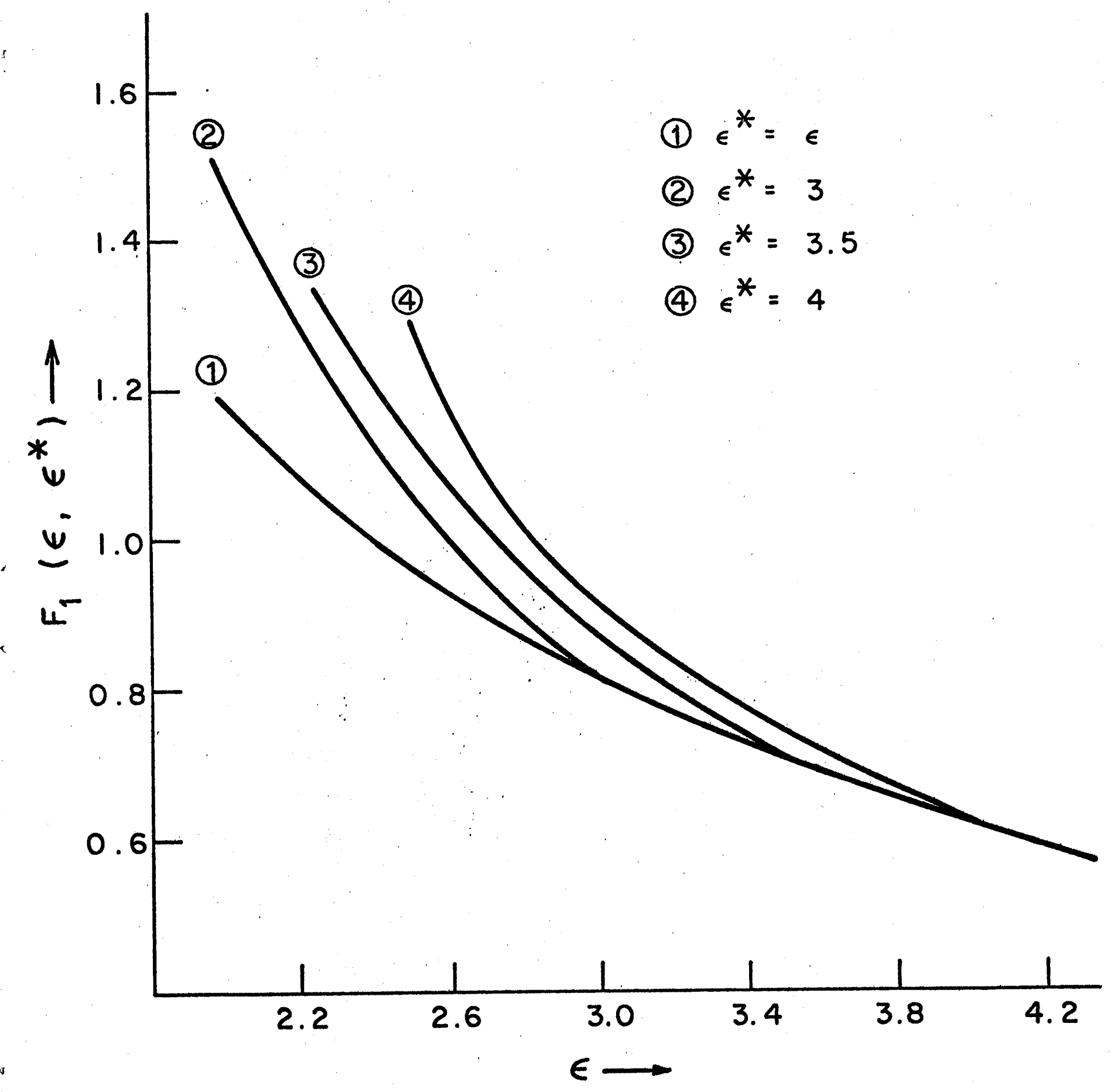

Figure 1. Graph of $F_{1}\left(\varepsilon, \varepsilon^{*}\right)$ vs, $\varepsilon$ 